\title{
Baryon Structure and the Chiral Symmetry of QCD
}

\author{
Leonid Ya. Glozman \\ Institut für Theoretische Physik, Universität Graz, \\ A-8010 Graz, Austria
}

\begin{abstract}
Beyond the spontaneous chiral symmetry breaking scale light and strange baryons should be considered as systems of three constituent quarks with an effective confining interaction and a chiral interaction that is mediated by the octet of Goldstone bosons (pseudoscalar mesons) between the constituent quarks.
\end{abstract}

\section{Introduction}

Our aim in physics is not only to calculate some observable and get a correct number but mainly to understand a physical picture responsible for the given phenomenon. It very often happens that a theory formulated in terms of fundamental degrees of freedom cannot answer such a question since it becomes overcomplicated at the related scale. Thus a main task in this case is to select those degrees of freedom which are indeed essential. For instance, the fundamental degrees of freedom in crystals are ions in the lattice, electrons and the electromagnetic field. Nevertheless, in order to understand electric conductivity, heat capacity, etc. we instead work with "heavy electrons" with dynamical mass, phonons and their interaction. In this case a complicated electromagnetic interaction of the electrons with the ions in the lattice is "hidden" in the dynamical mass of the electron and the interactions among ions in the lattice are eventually responsible for the collective excitations of the lattice - phonons, which are Goldstone bosons of the spontaneously broken translational invariance in the lattice of ions. As a result, the theory becomes rather simple - only the electron and phonon degrees of freedom and their interactions are essential for all the properties of crystals mentioned above.

Quite a similar situation takes place in QCD. One hopes that sooner or later one can solve the full nonquenched $\mathrm{QCD}$ on the lattice and get the correct nucleon and pion mass in terms of underlying degrees of freedom: current quarks and gluon fields. However, QCD at the scale of $1 \mathrm{GeV}$ becomes too complicated, and hence it is rather difficult to say in this case what kind of physics, inherent in QCD, is relevant to the nucleon mass and its lowenergy properties. In this lecture I will try to answer this question. I will

\footnotetext{
${ }^{0}$ Lecture given at the 35. Universitätswochen für Kern- und Teilchenphysik, Schladming, Austria, March 1996 (Perturbative and Nonperturbative Aspects of Quantum Field Theory, ed. by H. Latal and W. Schweiger, Springer 1996).
} 
show that it is the spontaneous breaking of chiral symmetry which is the most important QCD phenomenon in this case, and that beyond the scale of spontaneous breaking of chiral symmetry light and strange baryons can be viewed as systems of three constituent quarks which interact by the exchange of Goldstone bosons (pseudoscaler mesons) and are subject to confinement.

It is well known that at low temperature and density the approximate $S U(3)_{\mathrm{L}} \times S U(3)_{\mathrm{R}}$ chiral symmetry of QCD is realized in the hidden NambuGoldstone mode. The hidden mode of chiral symmetry is revealed by the existence of the octet of pseudoscalar mesons of low mass which represent the associated approximate Goldstone bosons. The $\eta^{\prime}$ (the $S U(3)$-singlet) decouples from the original nonet because of the $U(1)_{\mathrm{A}}$ anomaly [1], [2]. Another consequence of the spontaneous breaking of the approximate chiral symmetry of QCD is that the valence quarks acquire their dynamical or constituent mass [3], [4], [5], [6] through their interactions with the collective excitations of the QCD vacuum - the quark-antiquark excitations and the instantons.

We have recently suggested [7], [8] that beyond the scale of spontaneous breaking of chiral symmetry a baryon should be considered as a system of three constituent quarks with an effective quark-quark interaction that is formed by a central confining part and a chiral interaction mediated by the octet of pseudoscalar mesons between the constituent quarks.

The simplest representation of the most important component of the interaction of the constituent quarks mediated by the octet of pseudoscalar bosons in the $S U(3)_{\mathrm{F}}$ invariant limit is

$$
H_{\chi} \sim-\sum_{i<j} V\left(\mathbf{r}_{i j}\right) \boldsymbol{\lambda}_{i}^{\mathrm{F}} \cdot \boldsymbol{\lambda}_{j}^{\mathrm{F}} \boldsymbol{\sigma}_{i} \cdot \boldsymbol{\sigma}_{j} .
$$

Here $\left\{\boldsymbol{\lambda}_{i}^{\mathrm{F}}\right\}$ represents the flavor $S U(3)$ Gell-Mann matrices and the sums run over the constituent quarks.

Because of the flavor dependent factor $\boldsymbol{\lambda}_{i}^{\mathrm{F}} \cdot \boldsymbol{\lambda}_{j}^{\mathrm{F}}$ the chiral boson exchange interaction (1) will lead to orderings of the positive and negative parity states in the baryon spectra, which agree with the observed ones in all sectors. For the spectrum of the nucleon and $\Delta$ the strength of the chiral interaction between the constituent quarks is sufficient to shift the lowest positive parity states in the $N=2$ band (the $\mathrm{N}(1440)$ and $\Delta(1600)$ ) below the negative parity states in the $N=1$ band $(\mathrm{N}(1520)-\mathrm{N}(1535)$ and $\Delta(1620)-\Delta(1700))$. In the spectrum of the $\Lambda$, on the other hand, it is the negative parity flavor singlet states $(\Lambda(1405)-\Lambda(1520))$ that remain the lowest lying resonances, again in agreement with experiment. The mass splittings between the baryons with different strangeness, and between the $\Lambda$ and the $\Sigma$ which have identical flavor, spin and flavor-spin symmetries arise from the explicit breaking of the $S U(3)_{\mathrm{F}}$ symmetry that is caused by the mass splitting of the pseudoscalar meson octet, and the different masses of the $\mathrm{u}, \mathrm{d}$ and the $\mathrm{s}$ quarks.

This lecture has the following structure. Section 2 contains the proof why 
the commonly used perturbative gluon exchange interaction between the constituent quarks leads to incorrect ordering of positive and negative parity states in the spectra. In Section 3 we outline the importance of the spontaneous breaking of chiral symmetry for low-energy QCD, and in Section 4 we present a short historical sketch of the role of chiral symmetry in quark based models. Section 5 contains a description of the chiral boson exchange interaction (1). In Section 6 we describe the spectra of the nucleon, the $\Delta$ resonance and the $\Lambda$ hyperon as they are predicted by the $S U(3)_{\mathrm{F}}$ symmetric interaction (1). The effect of $S U(3)_{\mathrm{F}}$ breaking in the interaction is considered in Section 7. Section 8 is devoted to an "exact" three-body description of baryons where the interaction (1) is taken into account to all orders. In Section 9 we discuss the role of the exchange current corrections to the baryon magnetic moments that are associated with the pseudoscalar exchange interaction. Finally, in Section 10 some recent lattice - QCD results are discussed.

\section{Why the Gluon Exchange Bears no Relation to the Baryon Spectrum}

It was accepted by many people (but not by all) that the fine splittings in the baryon spectrum (in analogy to atomic physics they are often called "hyperfine splittings" as they arise from the spin-spin forces) are due to the gluon-exchange interaction between the constituent quarks [9], [10]. Now I shall address myself to a formal consideration why the one gluon exchange interaction cannot be relevant to the baryon spectrum.

The most important component of the one gluon exchange interaction [9] is the so called color-magnetic interaction

$$
H_{\mathrm{cm}} \sim-\alpha_{\mathrm{s}} \sum_{i<j} \frac{\pi}{6 m_{i} m_{j}} \boldsymbol{\lambda}_{i}^{\mathrm{C}} \cdot \boldsymbol{\lambda}_{j}^{\mathrm{C}} \boldsymbol{\sigma}_{i} \cdot \boldsymbol{\sigma}_{j} \delta\left(\mathbf{r}_{i j}\right),
$$

where $\left\{\boldsymbol{\lambda}_{i}^{\mathrm{C}}\right\}$ are color $S U(3)$ matrices. It is the permutational color-spin symmetry of the $3 q$ state which is mostly responsible for the contribution of the interaction (2). Indeed, the corresponding two-body matrix element is

$$
\begin{aligned}
<\left[f_{i j}\right]_{\mathrm{C}} \times\left[f_{i j}\right]_{\mathrm{S}}: & {\left[f_{i j}\right]_{\mathrm{CS}}\left|\boldsymbol{\lambda}_{i}^{\mathrm{C}} \cdot \boldsymbol{\lambda}_{j}^{\mathrm{C}} \boldsymbol{\sigma}_{i} \cdot \boldsymbol{\sigma}_{j}\right|\left[f_{i j}\right]_{\mathrm{C}} \times\left[f_{i j}\right]_{\mathrm{S}}:\left[f_{i j}\right]_{\mathrm{CS}}>} \\
& =\left\{\begin{array}{r}
8[11]_{\mathrm{C}},[11]_{\mathrm{S}}:[2]_{\mathrm{CS}} \\
-\frac{8}{3}[11]_{\mathrm{C}},[2]_{\mathrm{S}}:[11]_{\mathrm{CS}}
\end{array}\right.
\end{aligned}
$$

Thus the symmetrical color-spin pairs (i.e. with a $[2]_{\mathrm{CS}}$ Young pattern) experience an attractive contribution while the antisymmetrical ones $\left([11]_{\mathrm{CS}}\right)$ experience a repulsive contribution. Hence the color-magnetic contribution to the $\Delta$ state $\left([111]_{\mathrm{CS}}\right)$ is more repulsive than to the nucleon $\left([21]_{\mathrm{CS}}\right)$ and the $\Delta$ becomes heavier than the nucleon. The price is that $\alpha_{\mathrm{s}}$ should be larger than unity, which is bad. In addition there is no empirical indication in the 
spectrum for a large spin-orbit component of the gluon-exchange interaction [9] implied by this big value of $\alpha_{\mathrm{s}}$.

The crucial point is that the interaction (2) feels only colour and spin of the interacting quarks. Thus the structure of the $\mathrm{N}$ and $\Lambda$ spectra has to be the same as these baryons differ only by their flavour structure. If one looks at the Particle Data Group tables, however, one immediately sees a different ordering of the positive and negative parity states in both spectra. In the $\mathrm{N}$ spectrum the lowest states are $\frac{1}{2}^{+}, \mathrm{N}(939) ; \frac{1}{2}^{+}, \mathrm{N}(1440) ; \frac{1}{2}^{-}, \mathrm{N}(1535)-$ $\frac{3}{2}^{-}, \mathrm{N}(1520)$, while in the $\Lambda$ spectrum the ordering is as follows: $\frac{1}{2}^{+}, \Lambda(1115)$; $\frac{1}{2}^{-}, \Lambda(1405)-\frac{3}{2}^{-}, \Lambda(1520) ; \frac{1}{2}^{+}, \Lambda(1600)$. A weak flavor dependence via the quark masses in (2) cannot explain this paradox.

The interaction (2) cannot explain why the two-quantum excitations of positive parity $\mathrm{N}(1440), \Delta(1600), \Lambda(1600)$ and $\Sigma(1660)$ lie below the onequantum excitations of negative parity $\mathrm{N}(1535)-\mathrm{N}(1520), \Delta(1620)-\Delta(1700)$, $\Lambda(1670)-\Lambda(1690)$ and $\Sigma(1750)-\Sigma(?)$, respectively. For instance, the positive parity state $\mathrm{N}(1440)$ and the negative parity ones $\mathrm{N}(1535)-\mathrm{N}(1520)$ have the same mixed $\left([21]_{\mathrm{CS}}\right)$ color-spin symmetry thus the color-magnetic contribution to these states cannot be very different (a small difference is only due to the different radial structure of the positive and negative parity states). But the $\mathrm{N}(1440)$ state belongs to the $N=2$ shell, while the $\mathrm{N}(1535)-\mathrm{N}(1520)$ pair is a member of the $N=1$ band, which means that the $\mathrm{N}(1440)$ should lie approximately $\hbar \omega$ above the $\mathrm{N}(1535)-\mathrm{N}(1520)$. In the $\Delta$ spectrum the situation is even more dramatic. The $\Delta(1600)$ positive parity state has a completely antisymmetric CS-Young pattern $\left([111]_{\mathrm{CS}}\right)$, while the negative parity states $\Delta(1620)-\Delta(1700)$ have a mixed one. Thus the color-magnetic contribution to the $\Delta(1600)$ is much more repulsive than to the $\Delta(1620)-\Delta(1700)$. In addition the $\Delta(1600)$ is the $N=2$ state, while the pair $\Delta(1620)-\Delta(1700)$ belongs to the $N=1$ band. As a consequence the $\Delta(1600)$ must lie much higher than the $\Delta(1620)-\Delta(1700)$. All these features are well seen in the explicit 3-body calculations [11].

\section{Spontaneous Chiral Symmetry Breaking and its Consequences for Low-Energy QCD}

The QCD Lagrangian with three light flavors has a global symmetry

$$
S U(3)_{\mathrm{L}} \times S U(3)_{\mathrm{R}} \times U(1)_{\mathrm{V}} \times U(1)_{\mathrm{A}},
$$

if one neglects the masses of current $\mathrm{u}, \mathrm{d}$, and s quarks, which are small compared to a typical low-energy QCD scale of $1 \mathrm{GeV}$. The $U(1)_{\mathrm{A}}$ is not a symmetry at the quantum level due to the axial anomaly. If the $S U(3)_{\mathrm{L}} \times S U(3)_{\mathrm{R}}$ chiral symmetry of the QCD Lagrangian were intact in the vacuum state we would observe degenerate multiplets in the particle spectrum corresponding 
to the above chiral group, and all hadrons would have their degenerate partners with opposite parity. Since this does not happen the implication is that the chiral symmetry is spontaneously broken down to $S U(3)_{\mathrm{V}}$ in the QCD vacuum, i.e., realized in the hidden Nambu-Goldstone mode. A direct evidence for the spontaneously broken chiral symmetry is a nonzero value of the quark condensates for the light flavors

$$
<\operatorname{vacuum}|\bar{q} q| \text { vacuum }>\approx-(240-250 \mathrm{MeV})^{3},
$$

which represents the order parameter. That this is indeed so, we know from three independent sources: current algebra [12], QCD sum rules [13], and lattice gauge calculations [14]. There are two important generic consequences of the spontaneous chiral symmetry breaking. The first one is an appearance of the octet of pseudoscalar mesons of low mass, $\pi, \mathrm{K}, \eta$, which represent the associated approximate Goldstone bosons. The second one is that valence quarks acquire a dynamical or constituent mass. Both these consequences of the spontaneous chiral symmetry breaking are well illustrated by, e.g. the $\sigma$-model [15] or the Nambu and Jona-Lasinio model [16]. We cannot say at the moment for sure what the microscopical reason for spontaneous chiral symmetry breaking in the QCD vacuum is. It was suggested that this occurs when quarks propagate through instantons in the QCD vacuum [5], [6].

For the low-energy baryon properties it is only essential that beyond the spontaneous chiral symmetry breaking scale new dynamical degrees of freedom appear - constituent quarks and chiral fields. The low-energy baryon properties are mainly determined by these dynamical degrees of freedom and the confining interaction. This is quite in contrast to pseudoscalar mesons. In the chiral limit, $m_{\mathrm{u}}^{0}=m_{\mathrm{d}}^{0}=m_{\mathrm{s}}^{0}=0$, all members of the pseudoscalar octet $(\pi, \mathrm{K}, \eta)$ would have zero mass, which is most clearly seen in the Gell-MannOakes-Renner [12] relations

$$
\begin{aligned}
& m_{\pi^{0}}^{2}=-\frac{1}{f_{\pi}^{2}}\left(m_{\mathrm{u}}^{0}<\bar{u} u>+m_{\mathrm{d}}^{0}<\bar{d} d>\right)+O\left(m_{\mathrm{u}, \mathrm{d}}^{0}{ }^{2}\right), \\
& m_{\pi^{+,-}}{ }^{2}=-\frac{1}{f_{\pi}^{2}} \frac{m_{\mathrm{u}}^{0}+m_{\mathrm{d}}^{0}}{2}(<\bar{u} u>+<\bar{d} d>)+O\left(m_{\mathrm{u}, \mathrm{d}}^{0}{ }^{2}\right), \\
& m_{\mathrm{K}^{+,-}}{ }^{2}=-\frac{1}{f_{\pi}^{2}} \frac{m_{\mathrm{u}}^{0}+m_{\mathrm{s}}^{0}}{2}(<\bar{u} u>+<\bar{s} s>)+O\left({m_{\mathrm{u}, \mathrm{s}}^{0}}^{2}\right) \text {, } \\
& m_{\mathrm{K}^{0}, \overline{\mathrm{K}}^{0}}{ }^{2}=-\frac{1}{f_{\pi}^{2}} \frac{m_{\mathrm{d}}^{0}+m_{\mathrm{s}}^{0}}{2}(<\bar{d} d>+<\bar{s} s>)+O\left(m_{\mathrm{d}, \mathrm{s}}^{0}{ }^{2}\right), \\
& m_{\eta}{ }^{2}=-\frac{1}{3 f_{\pi}^{2}}\left(m_{\mathrm{u}}^{0}<\bar{u} u>+m_{\mathrm{d}}^{0}<\bar{d} d>+4 m_{\mathrm{s}}^{0}<\bar{s} s>\right)+O\left(m_{\mathrm{u}, \mathrm{d}, \mathrm{s}}^{0}{ }^{2}\right),
\end{aligned}
$$

that relate the pseudoscalar meson masses to the quark condensates and current quark masses $m^{0}$. Thus the nonzero masses of mesons are determined 
by the nonzero values of the current quark masses. In the baryon case, even in the chiral limit, baryons would have approximately their actual masses of the order of $1 \mathrm{GeV}$, as these masses are mostly determined by the dynamical (constituent) masses, the Goldstone boson exchange interaction among them, as well as a confining interaction. The dynamical (constituent) masses are in turn determined mainly by the quark condensates, which is most clearly seen from the gap equations of the Nambu and Jona-Lasinio model, and only weakly dependent on current quark masses. The role of the current quark masses in baryons is to break just the $S U(3)_{\mathrm{F}}$ symmetry in the baryon spectrum.

\section{Chiral Symmetry and the Quark Model (Historical Sketch)}

The importance of the constraints posed by chiral symmetry for the quark bag [17] and bag-like [18] models for the baryons has been recognized early. In bag models with restored chiral symmetry on the bag surface, or bag-like models, the massless current quarks within the bag were assumed to interact not only by perturbative gluon exchange but also through chiral meson field exchange. In these models the chiral field has the character of a compensating auxiliary field only, rather than a collective low frequency Goldstone quarkantiquark excitation. The possibility of a nonzero quark condensate was also not addressed. As it was discussed in a previous section, it is the quark condensate which is the most important characteristic determining the baryon properties. According to the bag philosophy, however, there is a perturbative QCD phase inside a bag, where all condensates vanish by definition. A general limitation of all bag and bag-like models is, of course, the lack of translational invariance, which is of crucial importance for a description of the excited states.

Common to these models is that the breaking of chiral symmetry arises from the confining interaction. This point of view contrasts with that of Manohar and Georgi [4], who pointed out that there should be two different scales in QCD with 3 flavors. At the first one of these, $\Lambda_{\chi \mathrm{SB}} \simeq 4 \pi f_{\pi} \simeq$ $1 \mathrm{GeV}$, the spontaneous breaking of the chiral symmetry occurs, and hence at distances beyond $\frac{1}{\Lambda_{\chi \mathrm{SB}}} \simeq 0.2 \mathrm{fm}$ the valence current quarks acquire their dynamical (constituent) mass (called "chiral quarks" in [4]), and the Goldstone bosons (mesons) appear. The other scale, $\Lambda_{\mathrm{QCD}} \simeq 100-300 \mathrm{MeV}$, is that which characterizes confinement, and the inverse of this scale roughly coincides with the linear size of a baryon. Between these two scales then the effective Lagrangian should be formed out of the gluon fields that provide a confining mechanism, as well as of the constituent quark and pseudoscalar meson fields. Manohar and Georgi did not, however, specify whether the baryons should be described as bound qqq states or as chiral solitons. 
The chiral symmetry breaking scale above fits well with that which appears in the instanton liquid picture of the QCD vacuum [5], [6]. In this model the quark condensates (i.e., equilibrium of virtual quark-antiquark pairs in

the vacuum state), as well as the gluon condensate, are supported by instanton fluctuations of a size $\sim 0.3 \mathrm{fm}$. Diakonov and Petrov [6] suggested that at low momenta (i.e., beyond the chiral symmetry breaking scale) QCD should be approximated by an effective chiral Lagrangian of the sigma-model type that contains valence quarks with dynamical (constituent) masses and meson fields. They considered a nucleon as three constituent quarks moving independently of one another in a self-consistent chiral field of the hedgehog form [19]. In this picture the $\Delta$ appears as a rotational excitation of the hedgehog, and no explicit confining interaction is included. A very similar description for the nucleon was suggested in [20], [21]. These types of models are now called "quark-soliton models" [22], [23].

The spontaneous breaking of chiral symmetry and its consequences - dynamical quark mass generation, appearance of the quark condensate, and pseudoscalar mesons as Goldstone excitations - are well illustrated by the Nambu and Jona-Lasinio model [16], [24]. This model lacks a confining interaction, which, as argued below, is essential for a realistic description of the properties of baryon physics.

\section{The Chiral Boson Exchange Interaction}

In an effective chiral description of the baryon structure, based on the constituent quark model, the coupling of the quarks and the pseudoscalar Goldstone bosons will (in the $S U(3)_{\mathrm{F}}$ symmetric approximation) have the form $i g \bar{\psi} \gamma_{5} \boldsymbol{\lambda}^{\mathrm{F}} \cdot \boldsymbol{\phi} \psi$ (or $\left.g /(2 m) \bar{\psi} \gamma_{\mu} \gamma_{5} \boldsymbol{\lambda}^{\mathrm{F}} \cdot \psi \partial^{\mu} \boldsymbol{\phi}\right)$, where $\psi$ is the fermion constituent quark field operator, $\phi$ the octet boson field operator, and $g$ is a coupling constant. A coupling of this form, in a nonrelativistic reduction for the constituent quark spinors, will - to lowest order - give rise to a Yukawa interaction between the constituent quarks, the spin-spin component of which has the form

$$
V_{\mathrm{Y}}\left(r_{i j}\right)=\frac{g^{2}}{4 \pi} \frac{1}{3} \frac{1}{4 m_{i} m_{j}} \boldsymbol{\sigma}_{i} \cdot \boldsymbol{\sigma}_{j} \boldsymbol{\lambda}_{i}^{\mathrm{F}} \cdot \boldsymbol{\lambda}_{j}^{\mathrm{F}}\left\{\mu^{2} \frac{e^{-\mu r_{i j}}}{r_{i j}}-4 \pi \delta\left(\mathbf{r}_{i j}\right)\right\} .
$$

Here $m_{i}$ and $m_{j}$ denote the masses of the interacting quarks, and $\mu$ that of the meson. There will also be an associated tensor component, which is discussed in ref. [8].

At short range the simple form (7) of the chiral boson exchange interaction cannot be expected to be realistic and should only be taken to be suggestive. Because of the finite spatial extent of both the constituent quarks and the pseudoscalar mesons the delta function in (7) should be replaced by a finite function, with a range of 0.6-0.7 fm, as suggested by the spatial extent of the mesons. In addition, the radial behaviour of the Yukawa potential (7) 
is valid only if the boson field satisfies a linear Klein-Gordon equation. The implications of the underlying chiral symmetry of QCD for the effective chiral Lagrangian (which in fact is not known), which contains constituent quarks as well as boson fields, are that these boson fields cannot be described by linear equations near their source. Therefore it is only at large distances, where the amplitude of the boson fields is small, that the quark-quark interaction reduces to the simple Yukawa form.

The latter point is rather important and has to be clarified. The radial dependence in (7) is a direct consequence of a free $\left(q^{2}-\mu^{2}\right)^{-1}$ Green function for the boson field, and a pseudoscalar $i g \bar{\psi} \gamma_{5} \lambda^{\mathrm{F}} \cdot \phi \psi$ or pseudovector $g /(2 m) \bar{\psi} \gamma_{\mu} \gamma_{5} \boldsymbol{\lambda}^{\mathrm{F}} \cdot \psi \partial^{\mu} \boldsymbol{\phi}$ coupling in both quark-meson vertices. Making use of the usual "static approximation" and neglecting the recoil corrections at both vertices in a nonrelativistic reduction for the constituent quark spinors, one arrives at (7). The free Green function above comes from the well-known Lagrangian for a free boson field $1 / 2 \partial_{\mu} \phi \partial^{\mu} \phi-1 / 2 \mu^{2} \phi^{2}$, which means that the boson field satisfies a linear Klein-Gordon equation.

On the other hand, the underlying $S U(3)_{\mathrm{R}} \times S U(3)_{\mathrm{L}}$ chiral symmetry of QCD tells that the Goldstone boson field cannot be described by such a simple Klein-Gordon Lagrangian. The transformation properties of the Goldstone boson field under $S U(3)_{\mathrm{R}}$ and $S U(3)_{\mathrm{L}}$ chiral rotations, $g_{\mathrm{R}}$ and $g_{\mathrm{L}}$, are well defined if the Goldstone boson fields $\phi$ are combined in a unitary matrix [25]

$$
\begin{gathered}
U(\phi)=\exp \left(i \frac{\lambda^{\mathrm{F}} \cdot \boldsymbol{\phi}}{f_{\pi}}\right), \\
U^{\prime}=g_{\mathrm{R}} U g_{\mathrm{L}}^{-1} .
\end{gathered}
$$

The "kinetic term" in this case is

$$
\frac{f_{\pi}^{2}}{4} \operatorname{Tr}\left(\partial_{\mu} U \partial^{\mu} U^{\dagger}\right),
$$

which in its expansion in powers of $\phi$ contains not only a free Klein-Gordon kinetic term but also other terms of higher powers. For instance, the terms of fourth order in the meson field give rise to $\pi-\pi$ scattering [26], etc. The full chiral Lagrangian describing the Goldstone boson field is unknown and should contain a quartic term involving derivatives $\partial_{\mu} U$ and higher order terms. It is clear that a dressed Green function for the field $\phi$ should be very different compared to the free Klein-Gordon Green function since the selfinteraction of the Goldstone boson field is important. Only far away from its source (the fermion current), where the amplitude of the boson field $\phi$ is small, the lowest term in powers of $\phi$ becomes dominant, and therefore only at large distances the quark-quark interaction reduces to its simple Yukawa form.

At this stage the proper procedure should be to avoid further specific assumptions about the short range behavior of $V(r)$ in (1), to extract instead 
the required matrix elements of it from the baryon spectrum, and to reconstruct by this an approximate radial form of $V(r)$. The overall minus sign in the effective chiral boson interaction in (1) corresponds to that of the short range term in the Yukawa interaction.

The flavor structure of the pseudoscalar octet exchange interaction in (1) between two quarks $i$ and $j$ should be understood as follows:

$$
\begin{aligned}
V\left(r_{i j}\right) & \lambda_{\mathbf{i}}^{\mathbf{F}} \cdot \lambda_{\mathbf{j}}^{\mathbf{F}} \boldsymbol{\sigma}_{i} \cdot \boldsymbol{\sigma}_{j} \\
& =\left(\sum_{a=1}^{3} V_{\pi}\left(r_{i j}\right) \lambda_{i}^{a} \lambda_{j}^{a}+\sum_{a=4}^{7} V_{\mathrm{K}}\left(r_{i j}\right) \lambda_{i}^{a} \lambda_{j}^{a}+V_{\eta}\left(r_{i j}\right) \lambda_{i}^{8} \lambda_{j}^{8}\right) \boldsymbol{\sigma}_{i} \cdot \boldsymbol{\sigma}_{j} .
\end{aligned}
$$

The first term in (11) represents the pion-exchange interaction, which acts only between light quarks. The second term represents the Kaon exchange interaction, which takes place in u-s and d-s pair states. The $\eta$-exchange, which is represented by the third term, is allowed in all quark pair states. In the $S U(3)_{\mathrm{F}}$ symmetric limit the constituent quark masses would be equal $\left(m_{\mathrm{u}}=m_{\mathrm{d}}=m_{\mathrm{s}}\right)$, the pseudoscalar octet would be degenerate and the mesonconstituent quark coupling constant would be flavor independent. In this limit the form of the pseudoscalar exchange interaction reduces to (1), which does not break the $S U(3)_{\mathrm{F}}$ invariance of the baryon spectrum. Beyond this limit the pion, Kaon and $\eta$ exchange interactions will differ $\left(V_{\pi} \neq V_{\mathrm{K}} \neq V_{\eta}\right)$, because of the difference between the strange and $\mathrm{u}$, d quark constituent masses $\left(m_{\mathrm{u}, \mathrm{d}} \neq m_{\mathrm{s}}\right)$, and because of the mass splitting within the pseudoscalar octet $\left(\mu_{\pi} \neq \mu_{\mathrm{K}} \neq \mu_{\eta}\right)$ (and possibly also because of flavor dependence in the mesonquark coupling constant). The source of both the $S U(3)_{\mathrm{F}}$ symmetry breaking constituent quark mass differences and the $S U(3)_{\mathrm{F}}$ symmetry breaking mass splitting of the pseudoscalar octet is the explicit chiral symmetry breaking in QCD.

\section{The Structure of the Baryon Spectrum}

The two-quark matrix elements of the interaction (1) are:

$$
\begin{gathered}
<\left[f_{i j}\right]_{\mathrm{F}} \times\left[f_{i j}\right]_{\mathrm{S}}:\left[f_{i j}\right]_{\mathrm{FS}}\left|-V\left(r_{i j}\right) \boldsymbol{\lambda}_{i}^{\mathrm{F}} \cdot \boldsymbol{\lambda}_{j}^{\mathrm{F}} \boldsymbol{\sigma}_{i} \cdot \boldsymbol{\sigma}_{j}\right|\left[f_{i j}\right]_{\mathrm{F}} \times\left[f_{i j}\right]_{\mathrm{S}}:\left[f_{i j}\right]_{\mathrm{FS}}> \\
=\left\{\begin{array}{c}
-\frac{4}{3} V\left(r_{i j}\right)[2]_{\mathrm{F}},[2]_{\mathrm{S}}:[2]_{\mathrm{FS}} \\
-8 V\left(r_{i j}\right)[11]_{\mathrm{F}},[11]_{\mathrm{S}}:[2]_{\mathrm{FS}} \\
4 V\left(r_{i j}\right)[2]_{\mathrm{F}},[11]_{\mathrm{S}}:[11]_{\mathrm{FS}} \\
\frac{8}{3} V\left(r_{i j}\right)[11]_{\mathrm{F}},[2]_{\mathrm{S}}:[11]_{\mathrm{FS}}
\end{array}\right.
\end{gathered}
$$

From these the following important properties may be inferred:

(i) At short range, where $V\left(r_{i j}\right)$ is positive, the chiral interaction (1) is attractive in the symmetric FS pairs and repulsive in the antisymmetric 
ones. At large distances the potential function $V\left(r_{i j}\right)$ becomes negative and the situation is reversed.

(ii) At short range, among the FS-symmetrical pairs, the flavor antisymmetric pairs experience a much larger attractive interaction than the flavorsymmetric ones, and among the FS-antisymmetric pairs the strength of the repulsion in flavor-antisymmetric pairs is considerably weaker than in the symmetric ones.

Given these properties we conclude, that with the given flavor symmetry, the more symmetrical the FS Young pattern is for a baryon the more attractive contribution at short range comes from the interaction (1). For two identical flavor-spin Young patterns $[f]_{\mathrm{FS}}$ the attractive contribution at short range is larger for the more antisymmetrical flavor Young pattern $[f]_{\mathrm{F}}$.

Consider first, for the purposes of illustration, a schematic model which neglects the radial dependence of the potential function $V(r)$ in (1), and assume a harmonic confinement among quarks as well as $m_{\mathrm{u}}=m_{\mathrm{d}}=m_{\mathrm{s}}$. In this model

$$
H_{\chi} \sim-\sum_{i<j} C_{\chi} \boldsymbol{\lambda}_{i}^{\mathrm{F}} \cdot \boldsymbol{\lambda}_{j}^{\mathrm{F}} \boldsymbol{\sigma}_{i} \cdot \boldsymbol{\sigma}_{j} .
$$

If the only interaction between the quarks were the flavor- and spinindependent harmonic confining interaction, the baryon spectrum would be organized in multiplets of the symmetry group $S U(6)_{\mathrm{FS}} \times U(6)_{\text {conf }}$. In this case the baryon masses would be determined solely by the orbital structure, and the spectrum would be organized in an alternative sequence of positive and negative parity states. The Hamiltonian (13), within a first order perturbation theory, reduces the $S U(6)_{\mathrm{FS}} \times U(6)_{\text {conf }}$ symmetry down to $S U(3)_{\mathrm{F}} \times S U(2)_{\mathrm{S}} \times U(6)_{\text {conf }}$, which automatically implies a splitting between the octet and decuplet baryons.

For the octet states $\mathrm{N}, \Lambda, \Sigma, \Xi(N=0$ shell, $N$ is the number of harmonic oscillator excitations in a 3-quark state) as well as for their first radial excitations of positive parity (breathing modes) N(1440), $\Lambda(1600), \Sigma(1660)$, $\Xi(?)(N=2$ shell $)$ the flavor and spin symmetries are $[3]_{\mathrm{FS}}[21]_{\mathrm{F}}[21]_{\mathrm{S}}$, and the contribution of the Hamiltonian (13) is $-14 C_{\chi}$. For the decuplet states $\Delta$, $\Sigma(1385), \Xi(1530), \Omega(N=0$ shell $)$ the flavor and spin symmetries, as well as the corresponding matrix element, are $[3]_{\mathrm{FS}}[3]_{\mathrm{F}}[3]_{\mathrm{S}}$ and $-4 C_{\chi}$, respectively. The first negative parity excitations $(N=1$ shell $)$ in the $\mathrm{N}$ and $\Sigma$ spectra $\mathrm{N}(1535)-\mathrm{N}(1520)$ and $\Sigma(1750)-\Sigma(?)$ are described by the $[21]_{\mathrm{FS}}[21]_{\mathrm{F}}[21]_{\mathrm{S}}$ symmetries, and the contribution of the interaction (13) in this case is $-2 C_{\chi}$. The first negative parity excitation in the $\Lambda$ spectrum $(N=1$ shell) $\Lambda(1405)$ $\Lambda(1520)$ is flavor singlet $[21]_{\mathrm{FS}}[111]_{\mathrm{F}}[21]_{\mathrm{S}}$, and, in this case, the corresponding matrix element is $-8 C_{\chi}$.

These matrix elements alone suffice to prove that the ordering of the lowest positive and negative parity states in the baryon spectrum will be correctly predicted by the chiral boson exchange interaction (13). The constant 
$C_{\chi}$ may be determined from the $\mathrm{N}-\Delta$ splitting to be $29.3 \mathrm{MeV}$. The oscillator parameter $\hbar \omega$, which characterizes the effective confining interaction, may be determined as one half of the mass differences between the first excited $\frac{1}{2}^{+}$ states and the ground states of the baryons, which have the same flavor-spin, flavor and spin symmetries (e.g. N(1440) - N, $\Lambda(1600)-\Lambda, \Sigma(1660)-\Sigma$ ), to be $\hbar \omega \simeq 250 \mathrm{MeV}$. Thus the two free parameters of this simple model are fixed and we can make now predictions. In the $\mathrm{N}$ and $\Sigma$ sectors the mass difference between the lowest excited $\frac{1}{2}^{+}$states $(\mathrm{N}(1440)$ and $\Sigma(1660))$ and $\frac{1}{2}^{-}-\frac{3}{2}^{-}$negative parity pairs $(\mathrm{N}(1535)-\mathrm{N}(1520)$ and $\Sigma(1750)-\Sigma(?))$ will then be

$$
\mathrm{N}, \Sigma: \quad m\left(\frac{1}{2}^{+}\right)-m\left(\frac{1}{2}^{-}-\frac{3}{2}^{-}\right)=250 \mathrm{MeV}-C_{\chi}(14-2)=-102 \mathrm{MeV},
$$

whereas for the $\Lambda$ system $(\Lambda(1600), \Lambda(1405)$ - $\Lambda(1520))$ it should be

$$
\Lambda: \quad m\left(\frac{1}{2}^{+}\right)-m\left(\frac{1}{2}^{-}-\frac{3}{2}^{-}\right)=250 \mathrm{MeV}-C_{\chi}(14-8)=74 \mathrm{MeV}
$$

This simple example shows how the chiral interaction (13) provides different ordering of the lowest positive and negative parity excited states in the spectra of the nucleon and the $\Lambda$-hyperon. This is a direct consequence of the symmetry properties of the boson-exchange interaction discussed at the beginning of this section. Namely, the $[3]_{\mathrm{FS}}$ state in the N(1440), $\Delta(1600)$ and $\Sigma(1660)$ positive parity resonances from the $N=2$ band feels a much stronger attractive interaction than the mixed symmetry state $[21]_{\mathrm{FS}}$ in the $\mathrm{N}(1535)-\mathrm{N}(1520), \Delta(1620)-\Delta(1700)$ and $\Sigma(1750)-\Sigma(?)$ resonances of negative parity $(N=1$ shell $)$. Consequently the masses of the positive parity states $\mathrm{N}(1440), \Delta(1600)$ and $\Sigma(1660)$ are shifted down relative to the other ones, which explains the reversal of the otherwise expected "normal ordering". The situation is different for $\Lambda(1405)-\Lambda(1520)$ and $\Lambda(1600)$, as the flavor state of $\Lambda(1405)-\Lambda(1520)$ is totally antisymmetric. Because of this the $\Lambda(1405)-\Lambda(1520)$ gains an attractive energy, which is comparable to that of the $\Lambda(1600)$, and thus the ordering suggested by the confining oscillator interaction is maintained.

Consider now, in addition, the radial dependence of the potential with the $S U(3)_{\mathrm{F}}$ invariant version (1) of the chiral boson exchange interaction (i.e., $\left.V_{\pi}(r)=V_{\mathrm{K}}(r)=V_{\eta}(r)\right)$. If the confining interaction in each quark pair is taken to have the harmonic oscillator form as above, the exact eigenvalues and eigenstates to the coinfining 3q Hamiltonian are

$$
\begin{gathered}
E=(N+3) \hbar \omega+3 V_{0}, \\
\Psi=\mid N(\lambda \mu) L[f]_{\mathrm{X}}[f]_{\mathrm{FS}}[f]_{\mathrm{F}}[f]_{\mathrm{S}}>,
\end{gathered}
$$

where $N$ is the number of quanta in the state, the Elliott symbol $(\lambda \mu)$ characterizes the $S U(3)$ harmonic oscillator symmetry, and $L$ is the orbital angular 
Table 1. The structure of the nucleon and $\Delta$ resonance states up to $N=2$, including 11 predicted unobserved or nonconfirmed states, indicated by question marks. The predicted energy values (in $\mathrm{MeV}$ ) are given in the brackets under the empirical ones.

\begin{tabular}{|c|c|c|c|}
\hline$N(\lambda \mu) L[f]_{\mathrm{X}}[f]_{\mathrm{FS}}[f]_{\mathrm{F}}[f]_{\mathrm{S}}$ & LS multiplet & $\begin{array}{l}\text { average } \\
\text { energy }\end{array}$ & $\delta M_{\chi}$ \\
\hline $0(00) 0[3]_{\mathrm{X}}[3]_{\mathrm{FS}}[21]_{\mathrm{F}}[21]_{\mathrm{S}}$ & $\frac{1}{2}^{+}, \mathrm{N}$ & 939 & $-14 P_{00}$ \\
\hline $0(00) 0[3]_{\mathrm{X}}[3]_{\mathrm{FS}}[3]_{\mathrm{F}}[3]_{\mathrm{S}}$ & $\frac{3}{2}^{+}, \Delta$ & $\begin{array}{l}1232 \\
\text { (input) }\end{array}$ & $-4 P_{00}$ \\
\hline $2(20) 0[3]_{\mathrm{X}}[3]_{\mathrm{FS}}[21]_{\mathrm{F}}[21]_{\mathrm{S}}$ & $\frac{1}{2}^{+}, \mathrm{N}(1440)$ & $\begin{array}{l}1440 \\
\text { (input) }\end{array}$ & $-7 P_{00}-7 P_{20}$ \\
\hline $1(10) 1[21]_{\mathrm{X}}[21]_{\mathrm{FS}}[21]_{\mathrm{F}}[21]_{\mathrm{S}}$ & $\frac{1}{2}^{-}, \mathrm{N}(1535) ; \frac{3}{2}^{-}, \mathrm{N}(1520)$ & $\begin{array}{l}1527 \\
\text { (input) }\end{array}$ & $-7 P_{00}+5 P_{11}$ \\
\hline $2(20) 0[3]_{\mathrm{X}}[3]_{\mathrm{FS}}[3]_{\mathrm{F}}[3]_{\mathrm{S}}$ & $\frac{3}{2}^{+}, \Delta(1600)$ & $\begin{array}{l}1600 \\
\text { (input) }\end{array}$ & $-2 P_{00}-2 P_{20}$ \\
\hline $1(10) 1[21]_{\mathrm{X}}[21]_{\mathrm{FS}}[3]_{\mathrm{F}}[21]_{\mathrm{S}}$ & $\frac{1}{2}^{-}, \Delta(1620) ; \frac{3}{2}^{-}, \Delta(1700)$ & $\begin{array}{l}1660 \\
(1719)\end{array}$ & $-2 P_{00}+6 P_{11}$ \\
\hline $1(10) 1[21]_{\mathrm{X}}[21]_{\mathrm{FS}}[21]_{\mathrm{F}}[3]_{\mathrm{S}}$ & $\begin{array}{l}\frac{1}{2}^{-}, \mathrm{N}(1650) ; \frac{3}{2}^{-}, \mathrm{N}(1700) \\
\frac{5}{2}^{-}, \mathrm{N}(1675)\end{array}$ & $\begin{array}{l}1675 \\
(1629)\end{array}$ & $-2 P_{00}+4 P_{11}$ \\
\hline $2(20) 2[3]_{\mathrm{X}}[3]_{\mathrm{FS}}[3]_{\mathrm{F}}[3]_{\mathrm{S}}$ & $\begin{array}{l}\frac{1}{2}^{+}, \Delta(1750 ?) ; \frac{3}{2}^{+}, \Delta(?) \\
\frac{5}{2}^{+}, \Delta(?) ; \frac{7}{2}^{+}, \Delta(?)\end{array}$ & $\begin{array}{l}1750 ? \\
(1675)\end{array}$ & $-2 P_{00}-2 P_{22}$ \\
\hline $2(20) 2[3]_{\mathrm{X}}[3]_{\mathrm{FS}}[21]_{\mathrm{F}}[21]_{\mathrm{S}}$ & $\frac{3}{2}^{+}, \mathrm{N}(1720) ; \frac{5}{2}^{+}, \mathrm{N}(1680)$ & $\begin{array}{l}1700 \\
\text { (input) }\end{array}$ & $-7 P_{00}-7 P_{22}$ \\
\hline $2(20) 0[21]_{\mathrm{X}}[21]_{\mathrm{FS}}[21]_{\mathrm{F}}[21]_{\mathrm{S}}$ & $\frac{1}{2}^{+}, \mathrm{N}(1710)$ & $\begin{array}{l}1710 \\
(1778)\end{array}$ & $-\frac{7}{2} P_{00}-\frac{7}{2} P_{20}+5 P_{11}$ \\
\hline $2(20) 0[21]_{\mathrm{X}}[21]_{\mathrm{FS}}[21]_{\mathrm{F}}[3]_{\mathrm{S}}$ & $\frac{3}{2}^{+}, \mathrm{N}(?)$ & $\begin{array}{l}? \\
(1813)\end{array}$ & $-P_{00}-P_{20}+4 P_{11}$ \\
\hline $2(20) 2[21]_{\mathrm{X}}[21]_{\mathrm{FS}}[21]_{\mathrm{F}}[21]_{\mathrm{S}}$ & $\frac{3}{2}^{+}, \mathrm{N}(1900 ?) ; \frac{5}{2}^{+}, \mathrm{N}(2000 ?)$ & $\begin{array}{l}1950 ? \\
(1909)\end{array}$ & $-\frac{7}{2} P_{00}-\frac{7}{2} P_{22}+5 P_{11}$ \\
\hline $2(20) 2[21]_{\mathrm{X}}[21]_{\mathrm{FS}}[21]_{\mathrm{F}}[3]_{\mathrm{S}}$ & $\begin{array}{l}\frac{1}{2}^{+}, \mathrm{N}(?) ; \frac{3}{2}^{+}, \mathrm{N}(?) \\
\frac{5}{2}^{+}, \mathrm{N}(?) ; \frac{7}{2}^{+}, \mathrm{N}(1990 ?)\end{array}$ & $\begin{array}{l}1990 ? \\
(1850)\end{array}$ & $-P_{00}-P_{22}+4 P_{11}$ \\
\hline $2(20) 0[21]_{\mathrm{X}}[21]_{\mathrm{FS}}[3]_{\mathrm{F}}[21]_{\mathrm{S}}$ & $\frac{1}{2}^{+}, \Delta(1910)$ & $\begin{array}{l}1910 \\
(1903)\end{array}$ & $-P_{00}-P_{20}+6 P_{11}$ \\
\hline $2(20) 2[21]_{\mathrm{X}}[21]_{\mathrm{FS}}[3]_{\mathrm{F}}[21]_{\mathrm{S}}$ & $\frac{3}{2}^{+}, \Delta(1920) ; \frac{5}{2}^{+}, \Delta(1905)$ & $\begin{array}{l}1912 \\
(1940)\end{array}$ & $-P_{00}-P_{22}+6 P_{11}$ \\
\hline
\end{tabular}

momentum. The spatial (X), flavor-spin (FS), flavor (F), and spin (S) permutational symmetries are indicated by corresponding Young patterns (diagrams) $[f]$. All these functions are well known (see, e.g., [27]). Note that the color state $[111]_{\mathrm{C}}$, which is common to all the states, has been suppressed in (17). By the Pauli principle $[f]_{\mathrm{X}}=[f]_{\mathrm{FS}}$.

The full Hamiltonian is the sum of the confining Hamiltonian and the 
Table 2. The structure of the $\Lambda$-hyperon states up to $N=2$, including predicted unobserved or nonconfirmed states, indicated by question marks. The predicted energies (in $\mathrm{MeV}$ ) are given in the brackets under the empirical values.

\begin{tabular}{|c|c|c|}
\hline$N(\lambda \mu) L[f]_{\mathrm{X}}[f]_{\mathrm{FS}}[f]_{\mathrm{F}}[f]_{\mathrm{S}} \quad$ LS multiplet & $\begin{array}{l}\text { average } \\
\text { energy }\end{array}$ & $\delta M_{\chi}$ \\
\hline $0(00) 0[3]_{\mathrm{X}}[3]_{\mathrm{FS}}[21]_{\mathrm{F}}[21]_{\mathrm{S}} \quad \frac{1}{2}^{+}, \Lambda$ & 1115 & $-14 P_{00}$ \\
\hline $1(10) 1[21]_{\mathrm{X}}[21]_{\mathrm{FS}}[111]_{\mathrm{F}}[21]_{\mathrm{S}} \frac{1}{2}^{-}, \Lambda(1405) ; \frac{3}{2}^{-}, \Lambda(1520)$ & $\begin{array}{l}1462 \\
(1512)\end{array}$ & $-12 P_{00}+4 P_{11}$ \\
\hline $2(20) 0[3]_{\mathrm{X}}[3]_{\mathrm{FS}}[21]_{\mathrm{F}}[21]_{\mathrm{S}}$ & $\begin{array}{l}1600 \\
(1616)\end{array}$ & $-7 P_{00}-7 P_{20}$ \\
\hline $1(10) 1[21]_{\mathrm{X}}[21]_{\mathrm{FS}}[21]_{\mathrm{F}}[21]_{\mathrm{S}} \frac{1}{2}^{-}, \Lambda(1670) ; \frac{3}{2}^{-}, \Lambda(1690)$ & $\begin{array}{l}1680 \\
(1703)\end{array}$ & $-7 P_{00}+5 P_{11}$ \\
\hline $\begin{array}{ll}1(10) 1[21]_{\mathrm{X}}[21]_{\mathrm{FS}}[21]_{\mathrm{F}}[3]_{\mathrm{S}} & \frac{1}{2}^{-}, \Lambda(1800) ; \frac{3}{2}^{-}, \Lambda(?) ; \\
& \frac{5}{2}^{-}, \Lambda(1830)\end{array}$ & $\begin{array}{l}1815 \\
(1805)\end{array}$ & $-2 P_{00}+4 P_{11}$ \\
\hline $2(20) 0[21]_{\mathrm{X}}[21]_{\mathrm{FS}}[111]_{\mathrm{F}}[21]_{\mathrm{S}} \frac{1}{2}^{+}, \Lambda(1810)$ & $\begin{array}{l}1810 \\
(1829)\end{array}$ & $-6 P_{00}-6 P_{20}+4 P_{11}$ \\
\hline$\frac{3}{2}^{+}, \Lambda(1890) ; \frac{5}{2}^{+}, \Lambda(1820)$ & $\begin{array}{l}1855 \\
(1878)\end{array}$ & $-7 P_{00}-7 P_{22}$ \\
\hline $2(20) 0[21]_{\mathrm{X}}[21]_{\mathrm{FS}}[21]_{\mathrm{F}}[21]_{\mathrm{S}} \frac{1}{2}^{+}, \Lambda(?)$ & $\begin{array}{l}? \\
(1954)\end{array}$ & $-\frac{7}{2} P_{00}-\frac{7}{2} P_{20}+5 P_{11}$ \\
\hline $2(20) 0[21]_{\mathrm{X}}[21]_{\mathrm{FS}}[21]_{\mathrm{F}}[3]_{\mathrm{S}}$ & $\begin{array}{l}? \\
(1989)\end{array}$ & $-P_{00}-P_{20}+4 P_{11}$ \\
\hline $\begin{array}{ll}2(20) 2[21]_{\mathrm{X}}[21]_{\mathrm{FS}}[21]_{\mathrm{F}}[3]_{\mathrm{S}} & \frac{1}{2}^{+}, \Lambda(?) ; \frac{3}{2}^{+}, \Lambda(?) ; \\
& \frac{5}{2}^{+} \Lambda(?) ; \frac{7}{2}^{+}, \Lambda(2020 ?)\end{array}$ & $\begin{array}{l}2020 ? \\
(2026)\end{array}$ & $-P_{00}-P_{22}+4 P_{11}$ \\
\hline $2(20) 2[21]_{\mathrm{X}}[21]_{\mathrm{FS}}[111]_{\mathrm{F}}[21]_{\mathrm{S}} \frac{3}{2}^{+}, \Lambda(?) ; \frac{5}{2}^{+}, \Lambda(?)$ & $\begin{array}{l}? \\
(2053)\end{array}$ & $-6 P_{00}-6 P_{22}+4 P_{11}$ \\
\hline $2(20) 2[21]_{\mathrm{X}}[21]_{\mathrm{FS}}[21]_{\mathrm{F}}[21]_{\mathrm{S}} \frac{3}{2}^{+}, \Lambda(?) ; \frac{5}{2}^{+}, \Lambda(2110)$ & $\begin{array}{l}2110 ? \\
(2085)\end{array}$ & $-\frac{7}{2} P_{00}-\frac{7}{2} P_{22}+5 P_{11}$ \\
\hline
\end{tabular}

chiral field interaction (1). When the boson exchange interaction (1) is treated in first order perturbation theory, the mass of the baryon states takes the form

$$
M=M_{0}+N \hbar \omega+\delta M_{\chi},
$$

where the chiral interaction contribution is $\delta M_{\chi}=\left\langle\Psi\left|H_{\chi}\right| \Psi\right\rangle$, and $M_{0}=$ $\sum_{i=1}^{3} m_{i}+3\left(V_{0}+\hbar \omega\right)$. The contribution from the chiral interaction to each baryon is a linear combination of the matrix elements of the two-body potential $V\left(r_{12}\right)$, defined as

$$
P_{n l}=<\varphi_{n l m}\left(\mathbf{r}_{12}\right)\left|V\left(r_{12}\right)\right| \varphi_{n l m}\left(\mathbf{r}_{12}\right)>.
$$

Here $\varphi_{n l m}\left(\mathbf{r}_{12}\right)$ represents the oscillator wavefunction with $n$ excited quanta. As we shall only consider the baryon states in the $N \leq 2$ bands, we shall only 
need the four radial matrix elements $P_{00}, P_{11}, P_{20}$ and $P_{22}$ for the numerical construction of the spectrum.

The contributions to all nucleon, $\Delta$ and $\Lambda$-hyperon states from the boson exchange interaction, in terms of the matrix elements $P_{n l}$, are listed in Tables 1 and 2 . In this approximate $S U(3)_{\mathrm{F}}$-invariant version of the chiral boson exchange interaction the $\Lambda-\mathrm{N}$ and the $\Xi-\Sigma$ mass differences would solely be ascribed to the mass difference between the s and u,d quarks, since all these baryons have identical orbital structure and permutational symmetries. The states in the $\Lambda$-spectrum would be degenerate with the corresponding states in the $\Sigma$-spectrum which have equal symmetries.

The oscillator parameter $\hbar \omega$ and the four integrals are extracted from the mass differences between the nucleon and the $\Delta(1232)$, the $\Delta(1600)$ and the $\mathrm{N}(1440)$, as well as the splittings between the nucleon and the average mass of the two pairs of states $\mathrm{N}(1535)-\mathrm{N}(1520)$ and $\mathrm{N}(1720)-\mathrm{N}(1680)$. This procedure yields the parameter values $\hbar \omega=157.4 \mathrm{MeV}, P_{00}=29.3 \mathrm{MeV}, P_{11}=45.2$ $\mathrm{MeV}, P_{20}=2.7 \mathrm{MeV}$ and $P_{22}=-34.7 \mathrm{MeV}$. Given these values, all other excitation energies (i.e., differences between the masses of given resonances and the corresponding ground states) of the nucleon, $\Delta$ - and $\Lambda$-hyperon spectra are predicted to within $\sim 15 \%$ of the empirical values where known, and are well within the uncertainty limits of those values. Note that these matrix elements provide a quantitatively satisfactory description of the $\Lambda$-spectrum even though they are extracted from the $\mathrm{N}-\Delta$ spectrum.

The relative magnitudes and signs of the numerical parameter values can be readily understood. If the potential function $V(\mathbf{r})$ is assumed to have the form of a Yukawa function with a smeared $\delta$-function term that is positive at short range $r \leq 0.6-0.7 \mathrm{fm}$, as suggested by the pion size $\sqrt{\left\langle r_{\pi}^{2}\right\rangle}=0.66$ fm, one expects $P_{20}$ to be considerably smaller than $P_{00}$ and $P_{11}$, as the radial wavefunction of the excited S-state has a node, and as it extends further into the region where the potential is negative. The negative value for $P_{22}$ is also natural, since the corresponding wavefunction is suppressed at short range and extends well beyond the expected node in the potential function.

\section{The $S U(3)_{F}$ Breaking Chiral Boson Interaction}

The model described above has relied on an interaction potential function $V(r)$ in (1) that is flavor independent. A refined version takes into account the explicit flavor dependence of the potential function in (11) $\left(V_{\pi} \neq V_{\mathrm{K}} \neq\right.$

$\left.V_{\eta}\right)$. In the following we show how this explicit flavor dependence provides an explanation of the mass splitting between the $\Lambda$ and the $\Sigma$ which have the same quark content and the same FS, F and S symmetries, i.e., they are degenerate within the $S U(3)_{\mathrm{F}}$ version (1) of the chiral boson exchange interaction.

Beyond the $S U(3)_{\mathrm{F}}$ limit the ground state baryons will be determined by the $\pi$-exchange radial integral $P_{00}^{\pi}$, the K-exchange one, $P_{00}^{\mathrm{K}}$, and by the 
$\eta$-exchange integrals, $P_{00}^{\mathrm{uu}}=P_{00}^{\mathrm{ud}}=P_{00}^{\mathrm{dd}}, P_{00}^{\mathrm{us}}$ and $P_{00}^{\mathrm{ss}}$, where the superscripts indicate quark pairs to which the $\eta$-exchange applies. As indicated by the Yukawa interaction (7) these matrix elements should be inversely proportional to the product of the quark masses of the pair state. Thus $P_{n l}^{\mathrm{us}}=\frac{m_{\mathrm{u}}}{m_{\mathrm{s}}} P_{n l}^{\mathrm{uu}}, \quad P_{n l}^{\mathrm{ss}}=\left(\frac{m_{\mathrm{u}}}{m_{\mathrm{s}}}\right)^{2} P_{n l}^{\mathrm{uu}}$. We also assume that $P_{00}^{\mathrm{us}} \simeq P_{00}^{\mathrm{K}}$, which is suggested by the fact that the quark masses are equal in the states in which these interactions act, and by the near equality of the Kaon and $\eta$ masses, $\mu_{\eta} \simeq \mu_{\mathrm{K}}$. Thus we have only two independent radial integrals.

To determine the integrals $P_{00}^{\pi}, P_{00}^{\mathrm{K}}$ and the quark mass difference $\Delta_{\mathrm{q}}=$ $m_{\mathrm{s}}-m_{\mathrm{u}}$ we consider the $\Sigma(1385)-\Sigma, \Delta-N$ and $\Lambda-N$ splittings:

$$
\begin{gathered}
m_{\Sigma(1385)}-m_{\Sigma}=4 P_{00}^{\mathrm{us}}+6 P_{00}^{\mathrm{K}}, \\
m_{\Delta}-m_{\mathrm{N}}=12 P_{00}^{\pi}-2 P_{00}^{\mathrm{uu}}, \\
m_{\Lambda}-m_{\mathrm{N}}=6 P_{00}^{\pi}-6 P_{00}^{\mathrm{K}}+\Delta_{\mathrm{q}},
\end{gathered}
$$

which imply $P_{00}^{\mathrm{K}}=19.6 \mathrm{MeV}, \Delta_{\mathrm{q}}=121 \mathrm{MeV}$, if the conventional value of 340 $\mathrm{MeV}$ is given to $m_{\mathrm{u}}, P_{00}^{\pi}=28.9 \mathrm{MeV}$ and the quark mass ratio $m_{\mathrm{s}} / m_{\mathrm{u}}=1.36$. These values of the matrix elements lead to the values of $65 \mathrm{MeV}$ and 139 $\mathrm{MeV}$ for the $\Sigma-\Lambda$ and the $\Xi-\Sigma$ mass differences

$$
\begin{gathered}
m_{\Sigma}-m_{\Lambda}=8 P_{00}^{\pi}-4 P_{00}^{\mathrm{K}}-\frac{4}{3} P_{00}^{\mathrm{uu}}-\frac{8}{3} P_{00}^{\mathrm{us}}, \\
m_{\Xi}-m_{\Sigma}=P_{00}^{\pi}+\frac{1}{3} P_{00}^{\mathrm{uu}}-\frac{4}{3} P_{00}^{\mathrm{ss}}+\Delta_{\mathrm{q}}
\end{gathered}
$$

in good agreement with the empirical values of $77 \mathrm{MeV}$ and $125 \mathrm{MeV}$, respectively.

A description of the other parts of the $\Sigma, \Xi$ and $\Omega$ spectra can be found in $[8]$.

\section{Three-Body Faddeev Calculations}

In the previous sections we have shown how the Goldstone boson exchange (GBE), taken to first order perturbation theory and without explicit parameterizing the radial dependence, can explain the correct level ordering of positive and negative parity states in light and strange baryon spectra, as well as the splittings in those spectra. A question, however, arises about what will happen beyond first order perturbation theory. In order to check this we have numericaly solved three-body Faddeev equations [28]. Besides the confinement potential, which is now taken in linear form, the GBE interaction between the constituent quarks is now included to all orders. These results further support the adequacy of the GBE for baryon spectroscopy.

In addition to the octet-exchange interaction we include here also the flavor-singlet $\left(\eta^{\prime}\right)$ exchange. In the large $N_{\mathrm{C}}$ limit the axial anomaly becomes 
suppressed [29], and the $\eta^{\prime}$ becomes the ninth Goldstone boson of the spontaneously broken $U(3)_{\mathrm{L}} \times U(3)_{\mathrm{R}}$ chiral symmetry in addition to the octet of pseudoscalar mesons [30].

For the GBE the spin-spin component of the interaction between the constituent quarks $i$ and $j$ reads:

$$
\begin{aligned}
V_{\chi}\left(\mathbf{r}_{i j}\right) & =\left\{\sum_{a=1}^{3} V_{\pi}\left(\mathbf{r}_{i j}\right) \lambda_{i}^{a} \lambda_{j}^{a}\right. \\
& \left.+\sum_{a=4}^{7} V_{\mathrm{K}}\left(\mathbf{r}_{i j}\right) \lambda_{i}^{a} \lambda_{j}^{a}+V_{\eta}\left(\mathbf{r}_{i j}\right) \lambda_{i}^{8} \lambda_{j}^{8}+V_{\eta^{\prime}}\left(\mathbf{r}_{i j}\right) \lambda_{i}^{0} \lambda_{j}^{0}\right\} \boldsymbol{\sigma}_{i} \cdot \boldsymbol{\sigma}_{j},
\end{aligned}
$$

where $\lambda^{a}, a=1, \ldots, 8$ are flavor Gell-Mann matrices and $\lambda^{0}=\sqrt{2 / 3} 11$.

In the simplest case, when the boson field satisfies the linear Klein-Gordon equation, one has the following spatial dependence for the meson-exchange potentials in (25):

$$
\begin{gathered}
V_{\gamma}\left(\mathbf{r}_{i j}\right)=\frac{g_{\gamma}^{2}}{4 \pi} \frac{1}{3} \frac{1}{4 m_{i} m_{j}}\left\{\mu_{\gamma}^{2} \frac{e^{-\mu_{\gamma} r_{i j}}}{r_{i j}}-4 \pi \delta\left(\mathbf{r}_{i j}\right)\right\}, \\
\left(\gamma=\pi, \mathrm{K}, \eta, \eta^{\prime}\right),
\end{gathered}
$$

with quark and meson masses $m_{i}$ and $\mu_{\gamma}$, respectively.

Eq. (26) contains both the traditional long-range Yukawa potential as well as a $\delta$-function term. It is the latter that is of crucial importance for baryon physics. We already discussed in Section 5 that it is strictly valid only for pointlike particles, and that it must be smeared out since the constituent quarks and pseudoscalar mesons have finite size, and in addition the boson fields in a chiral Lagrangian should in fact satisfy a nonlinear equation. Furthermore it is quite natural to assume that at distances $r \ll r_{0}$, where $r_{0}$ can be related to the constituent quark and pseudoscalar meson sizes, there is no chiral boson-exchange interaction, since this is the region of perturbative QCD with the original QCD degrees of freedom. The interactions at these very short distances are not essential for the low-energy properties of baryons. Consequently we use a two-parameter "representation" for the $\delta$-function term in (26)

$$
4 \pi \delta\left(\mathbf{r}_{i j}\right) \Rightarrow \frac{4}{\sqrt{\pi}} \alpha^{3} \exp \left(-\alpha^{2}\left(r-r_{0}\right)^{2}\right) .
$$

Following the arguments above one should also cut off the Yukawa part of the GBE for $r<r_{0}$.

The $\pi \mathrm{q}$ coupling constant can be extracted from the phenomenological pion-nucleon coupling [8] as $\frac{g_{8}^{2}}{4 \pi}=0.67$. For simplicity (and to avoid any 
additional free parameter), the same coupling constant is assumed for the coupling between the $\eta$ - meson and the constituent quark. This is exactly in the spirit of unbroken $S U(3)_{\mathrm{F}}$ symmetry. For the flavor-singlet $\eta^{\prime}$, however, we must take a different coupling $\frac{g_{0}^{2}}{4 \pi}$, as the $\eta^{\prime}$ decouples from the pseudoscalar octet due to the $U(1)_{\mathrm{A}}$ anomaly. This fact is illustrated best by the failure of the Gell-Mann-Oakes-Renner relations [12] for the flavor singlet [1]. Lacking a phenomenological value, we treat $g_{0}^{2} / 4 \pi$ as a free parameter. The constituent masses of the $\mathrm{u}$ and d quarks are taken to be $340 \mathrm{MeV}$, as suggested by the nucleon magnetic moments.

In the present calculation we neglect tensor meson-exchange forces. We expect their role to be of minor importance for the main features of the baryon spectra [8] (mainly due to the absence of the strong $\delta$-function part in this case).

Our full interquark potential is thus given by

$$
V\left(\mathbf{r}_{i j}\right)=V_{\chi}^{\text {octet }}\left(\mathbf{r}_{i j}\right)+V_{\chi}^{\text {singlet }}\left(\mathbf{r}_{i j}\right)+C r_{i j} .
$$

While all masses and the octet coupling constant are predetermined, we treated $r_{0}, \alpha,\left(g_{0} / g_{8}\right)^{2}$, and $C$ as free parameters and determined their values to be:

$$
r_{0}=0.43 \mathrm{fm}, \alpha=2.91 \mathrm{fm}^{-1},\left(g_{0} / g_{8}\right)^{2}=1.8, C=0.474 \mathrm{fm}^{-2} .
$$

Notice that we do not need any constant $V_{0}$, which is usually added to the confining potential. In fact only four free parameters suffice to describe all 14 lowest states of the $\mathrm{N}$ and $\Delta$ spectra, including the absolute value of the nucleon (ground state). At the present stage of determining the qq potential due to GBE we were led by the principle of working with the smallest possible number of free parameters. Therefore we took the octet coupling constant $g_{8}^{2} / 4 \pi$, and likewise the constituent quark mass $m$, as predetermined. Of course, one may expect that subsequent studies within the GBE model will put further constraints on the parametrization of the $q q$ potential.

The qq potential (28) constitutes the dynamical input into our 3-body Faddeev calculations of the baryon spectra. We show our results in Fig. 1 for the parametrization of the qq interaction as given above. It is well seen that the whole set of lowest $\mathrm{N}$ and $\Delta$ states is reproduced quite correctly. In the most unfavourable cases deviations from the experimental values do not exceed $3 \%$ ! In addition all level orderings are correct. In particular, the positiveparity state $\mathrm{N}(1440)$ (Roper resonance) lies below the pair of negative-parity states $\mathrm{N}(1535)-\mathrm{N}(1520)$. The same is true in the $\Delta$ spectrum with $\Delta(1600)$ and the pair $\Delta(1620)-\Delta(1700)$. We emphasize again that the qq potential (28) is able to predict also the absolute value of the nucleon mass. In previous models an arbitrary constant was usually needed to achieve the correct value of $939 \mathrm{MeV}$. 


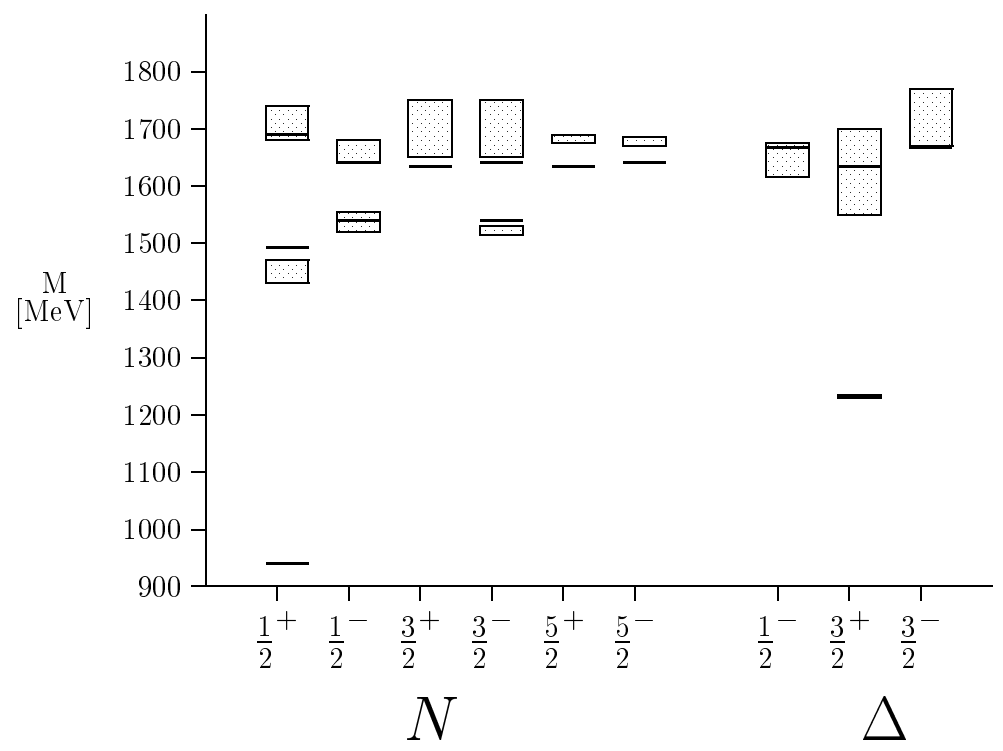

Fig. 1. Energy levels for the 14 lowest non-strange baryons with total angular momentum and parity $J^{P}$. The shadowed boxes represent experimenta uncertainties.

At the present stage of our investigation of the baryon spectra with the GBE interaction we have left out the tensor forces. Therefore the finestructure splittings in the $L S$-multiplets are not yet introduced. However, it is clear from the observed smallness of these splittings and from the arguments given above, that the tensor component of the GBE can play only a minor role. Here we also note that the Yukawa part of the interaction in (26) is only of secondary importance. In fact, the pattern of Fig. 1 could also be described with the " $\delta$-part" (27) alone (and a slightly modified set of parameters).

It is instructive to learn how the GBE affects the energy levels when it is switched on and its strength (coupling constant) is gradually increased (Fig. 2 ). Starting out from the case with confinement only, one observes that the degeneracy of states is removed and an inversion of the ordering of positiveand negative-parity states is achieved, both in the $\mathrm{N}$ and $\Delta$ excitations. From Fig. 2 also the crucial importance of the chiral interaction $V_{\chi}$ becomes evident. Notice that the strength of our confinement, $C=0.474 \mathrm{fm}^{-2}$, is rather small and the confining interaction contributes much less to the splittings than the GBE. The relative "weakness" of our effective confining interaction could be due to a partial cancellation between the much stronger color-electric confinement and the $\sigma$-exchange since they are of opposite sign. Due to the 


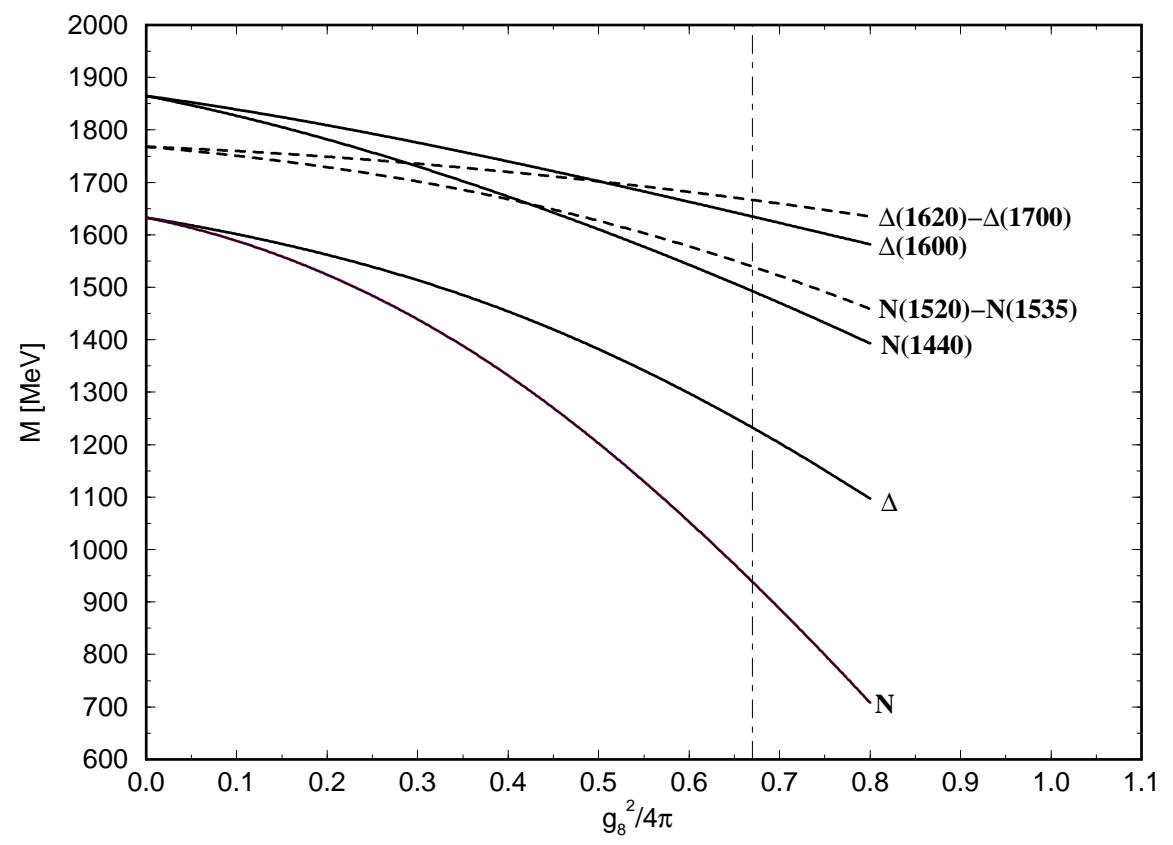

Fig. 2. Level shifts of some lowest baryons as a function of the strength of the GBE. Solid and dashed lines correspond to positive- and negative-parity states, respectively.

same reason one cannot expect that the effective confining interaction between the constituent quarks is strictly of linear form.

\section{Exchange Current Corrections} to the Magnetic Moments

A flavor dependent interaction of the form (1) will imply the presence of an irreducible two-body exchange current operator, as seen, e.g., directly from the continuity equation, by which the commutator of the interaction and the single particle charge operator is equal to the divergence of the exchange current density [31]. 
The general form of the octet vector exchange current operator, that is associated with the complete octet mediated interaction (11), is [8]

$$
\boldsymbol{\mu}^{\mathrm{ex}}=\mu_{\mathrm{N}}\left\{\tilde{V}_{\pi}\left(r_{i j}\right)\left(\lambda_{i}^{1} \lambda_{j}^{2}-\lambda_{i}^{2} \lambda_{j}^{1}\right)+\tilde{V}_{\mathrm{K}}\left(r_{i j}\right)\left(\lambda_{i}^{4} \lambda_{j}^{5}-\lambda_{i}^{5} \lambda_{j}^{4}\right)\right\}\left(\boldsymbol{\sigma}_{i} \times \boldsymbol{\sigma}_{j}\right) .
$$

Here $\tilde{V}_{\pi}(r)$ and $\tilde{V}_{\mathrm{K}}(r)$ are dimensionless functions that describe $\pi$ and $K$ exchange, respectively, and which include both the pionic (kaonic) current and the pair current term at long range.

Consider a simplified nonrelativistic constituent quark model. The impulse approximation expressions for the magnetic moments of the ground state octet baryons and their experimental values are listed in Table 3 (columns "IA" and "exp", respectively). A natural approach is to determine the mass ratios $m_{\mathrm{N}} / m_{\mathrm{u}}$ and $m_{\mathrm{N}} / m_{\mathrm{S}}$ to fit the experimental values of the magnetic moments of the $\Sigma^{-}$and $\Xi^{-}$octet and the $\Omega$ and $\Delta^{++}\left(\mu_{\Omega}=-2.019 \pm 0.054 \mu_{\mathrm{N}}\right.$, $\mu_{\Delta^{+}+}=4.52 \pm 0.50 \mu_{\mathrm{N}}$ ) decuplet baryons, which are unaffected by the exchange current operator (29). While with only two independent variables it is not possible to fit all four experimental magnetic moments exactly, the best overall fit, $\mu_{\Sigma^{-}}=-1.00 \mu_{\mathrm{N}}, \mu_{\Xi^{-}}=-0.59 \mu_{\mathrm{N}}, \mu_{\Omega^{-}}=-2.01 \mu_{\mathrm{N}}, \mu_{\Delta^{++}}=$ $5.52 \mu_{\mathrm{N}}$, happens to be obtained with precisely the ratios $m_{\mathrm{N}} / m_{\mathrm{u}}=2.76$ and $m_{\mathrm{N}} / m_{\mathrm{s}}=2.01$, which were used for the constituent quark masses to fit ground state baryons $\left(m_{\mathrm{u}}=340 \mathrm{MeV}\right.$ and $\left.m_{\mathrm{s}}=467 \mathrm{MeV}\right)$.

We find (see Table 3 ) that the meson exchange current contributions systematically improve the predictions of the naive constituent quark model (i.e., with one-body quark currents only) for all known magnetic moments.

As the constituent quarks are not too heavy, both their electromagnetic and axial current operators have significant relativistic correction terms. Their effect is to reduce the magnitude of the predicted values of both the axial coupling constants and the magnetic moments of the baryons that are given by the static quark model. This correction reduces the standard overprediction of the axial current coupling constant of the nucleon (5/3 vs 1.24$)$ and the strange baryons, but it worsens the mostly satisfactory predictions for the magnetic moments of the baryons that are obtained with the static quark model. In ref. [32] it is shown that the exchange current corrections associated with the chiral boson exchange interaction between the quarks can compensate for the relativistic correction in the latter case, while leaving it operative in the case of the axial coupling constants. This then makes it possible to obtain at least qualitatively satisfactory simultaneous description of both the magnetic moments and the axial coupling constants.

\section{Instead of a Conclusion}

Instead of a conclusion we discuss some important recent lattice QCD results in this last section. It was shown already a few years ago that one can obtain a qualitatively correct splitting between $\Delta$ and $\mathrm{N}$ already within a 
Table 3. Magnetic moments of the baryon octet (in nuclear magnetons). Column IA contains the quark model impulse approximation expressions, column "exp" the experimental values, column I the impulse approximation predictions, column II the exchange current contribution with $\left\langle\varphi_{000}\left(\mathbf{r}_{12}\right)\left|\tilde{V}_{\pi}\left(r_{12}\right)\right| \varphi_{000}\left(\mathbf{r}_{12}\right)\right\rangle=-0.018$ and $<\varphi_{000}\left(\mathbf{r}_{12}\right)\left|\tilde{V}_{\mathrm{K}}\left(r_{12}\right)\right| \varphi_{000}\left(\mathbf{r}_{12}\right)>=0.03$, and column III the net predictions.

\begin{tabular}{|l|l|l|l|l|l|}
\hline & IA & exp & I & II & III \\
\hline $\mathrm{p}$ & $\frac{m_{\mathrm{N}}}{m_{\mathrm{u}}}$ & +2.79 & +2.76 & +0.07 & +2.83 \\
$\Lambda$ & $-\frac{2}{3} \frac{m_{\mathrm{N}}}{m_{\mathrm{u}}}$ & -1.91 & -1.84 & -0.07 & -1.91 \\
$\Sigma^{+}$ & $-\frac{1}{3} \frac{m_{\mathrm{N}}}{m_{\mathrm{s}}}$ & -0.61 & -0.67 & +0.06 & -0.61 \\
$\frac{8}{9} \frac{m_{\mathrm{N}}}{m_{\mathrm{u}}}+\frac{1}{9} \frac{m_{\mathrm{N}}}{m_{\mathrm{s}}}$ & +2.42 & +2.68 & -0.12 & +2.56 \\
$\Sigma^{0}$ & $\frac{2}{9} \frac{m_{\mathrm{N}}}{m_{\mathrm{u}}}+\frac{1}{9} \frac{m_{\mathrm{N}}}{m_{\mathrm{s}}}$ & $?$ & +0.84 & -0.06 & +0.72 \\
$\Sigma^{0} \rightarrow \Lambda$ & $-\frac{1}{\sqrt{3}} \frac{m_{\mathrm{N}}}{m_{\mathrm{u}}}$ & $|1.61|$ & -1.59 & -0.01 & -1.60 \\
$\Sigma^{-}$ & $-\frac{4}{9} \frac{m_{\mathrm{N}}}{m_{\mathrm{u}}}+\frac{1}{9} \frac{m_{\mathrm{N}}}{m_{\mathrm{s}}}$ & -1.16 & -1.00 & 0 & -1.00 \\
$\Xi^{0}$ & $-\frac{2}{9} \frac{m_{\mathrm{N}}}{m_{\mathrm{u}}}-\frac{4}{9} \frac{m_{\mathrm{N}}}{m_{\mathrm{s}}}$ & -1.25 & -1.51 & +0.12 & -1.39 \\
$\Xi^{-}$ & $\frac{1}{9} \frac{m_{\mathrm{N}}}{m_{\mathrm{u}}}-\frac{4}{9} \frac{m_{\mathrm{N}}}{m_{\mathrm{s}}}$ & -0.65 & -0.59 & 0 & -0.59 \\
\hline
\end{tabular}

quenched approximation (for a review and references see [33]). Within the quenched approximation to QCD the sea quark closed loop diagrams generated by gluon lines are neglected. Thus in the quenched approximation for baryons one takes into account only 3 continuous valence quark lines and full gluodynamics. This quenched approximation contains, however, part of antiquark effects related to the $\mathrm{Z}$ graphs formed of valence quark lines. One can even construct diagrams within the quenched approximation which correspond to the exchange of the color-singlet isospin 1 or $0 \mathrm{q} \overline{\mathrm{q}}$ pairs between valence quark lines [34]. It is also important that these diagrams contribute to the baryon mass to leading order $\left(\sim N_{\mathrm{C}}\right)$ in a $1 / N_{\mathrm{C}}$ expansion [35] (their contribution to the $\Delta-\mathrm{N}$ splitting appears, however, to subleading orders).

From the quenched measurements [33] it is not clear what were the physical reason for the $\Delta-\mathrm{N}$ splitting: gluon exchanges, instantons, or something else. To clarify this question, Liu and Dong have recently measured the $\Delta-\mathrm{N}$ splitting in the quenched and a further so-called "valence approximation" [36]. In the valence approximation the quarks are limited to propagating only forward in time (i.e., Z graphs and related quark-antiquark 
pairs are removed). The gluon exchange and all other possible gluon configurations, including instantons, are exactly the same in both approximations. The striking result is that the $\Delta-\mathrm{N}$ splitting is observed only in the quenched approximation but not in the valence approximation, in which the $\mathrm{N}$ and the $\Delta$ levels are degenerate within error bars. Consequently the $\Delta-\mathrm{N}$ splitting must receive a considerable contribution from the diagrams with q $\bar{q}$ excitations, which correspond to the meson exchanges, but not from the gluon exchange or instanton-induced interaction between quarks (to be precise, the instanton-induced interaction could be rather important for the interactions between quarks and antiquarks).

If the observation of Liu and Dong is confirmed it would be important to measure the relative positions of the lowest excited states of positive and negative parity in the $\mathrm{N}, \Delta, \Lambda$ and $\Sigma$ spectra within both the quenched and the valence approximation. One expects that, if the entire $\Delta-\mathrm{N}$ splitting (i.e., $300 \mathrm{MeV}$ ) is due to the antiquark excitations in the quenched approximation, then $1 / 2^{+}, \mathrm{N}(1440)$ should be below the negative parity pair $1 / 2^{-}, \mathrm{N}(1535)-$ $3 / 2^{-}, \mathrm{N}(1520)$, while in the $\Lambda$ spectrum the situation should be opposite: the negative parity pair $1 / 2^{-}, \Lambda(1405)-3 / 2^{-}, \Lambda(1520)$ should be below the first positive parity excitation $1 / 2^{+}, \Lambda(1600)$. In the valence approximation the spin-spin force among quarks, which is due to Goldstone boson exchange, is absent, and the relative position of $1 / 2^{+}, \mathrm{N}(1440)$ and $1 / 2^{-}, \mathrm{N}(1535)-$ $3 / 2^{-}, \mathrm{N}(1520)$ should be just opposite to the quenched approximation: the $1 / 2^{+}, \mathrm{N}(1440)$ should be above the negative parity pair. However, in the $\Lambda$ spectrum the negative parity pair $1 / 2^{-}, \Lambda(1405)-3 / 2^{-}, \Lambda(1520)$ should still be below the $1 / 2^{+}, \Lambda(1600)$.

\section{Acknowledgements}

It is a pleasure to thank Dan Riska, Zoltan Papp and Willi Plessas for their collaboration that was crucial for the results presented in this lecture.

\section{References}

1. S. Weinberg, Phys. Rev. D11 (1975) 3583.

2. G.'t Hooft, Phys. Rev. D14 (1976) 3432.

3. S. Weinberg, Physica 96 A (1979) 327.

4. A. Manohar and H. Georgi, Nucl. Phys. B234 (1984) 189.

5. E.V. Shuryak, Phys.Rep. C115 (1984) 152.

6. D. I. Diakonov and V. Yu. Petrov, Phys. Lett. B147 (1984) 351; Nucl. Phys. B272 (1986) 457.

7. L. Ya. Glozman and D. O. Riska, The Baryon Spectrum and Chiral Dynamics, Preprint HU-TFT-94-47, [LANL hep-ph 9411279] (1994); Systematics of the Light and Strange Baryons and the Symmetries of QCD, Preprint HU-TFT-9448, [LANL hep-ph 9412231] (1994).

8. L. Ya. Glozman and D. O. Riska, Physics Reports 268 (1996) 263. 
9. A. DeRujula, H. Georgi, and S. L. Glashow, Phys. Rev. D12 (1975) 147.

10. N. Isgur and G. Karl, Phys. Rev. D18 (1978) 4187; D19 (1979) 2653.

11. B. Silvestre-Brac and C. Gignoux, Phys. Rev. D32 (1985) 743.

12. M. Gell-Mann, R. J. Oakes, and B. Renner, Phys. Rev. 175 (1968) 2195.

13. M. Shifman, A. Vainstein, and V. Zahkarov, Nucl. Phys. B147 (1979) 385, 448.

14. D. R. Daniel et al, Phys. Rev. D46 (1992) 3130; M. N. Fukugita et al, Phys. Rev. D47 (1993) 4739.

15. M. Gell-Mann and M. Levy, Nuovo Cim. 16 (1960) 705.

16. Y. Nambu and G. Jona-Lasinio, Phys. Rev. 122 (1961) 345.

17. A. Chodos and C. B. Thorn, Phys. Rev.D12 (1975) 2733; G. E. Brown and M. Rho, Phys. Lett. B82 (1979) 177; A. W. Thomas, Adv. Nucl. Phys. 13 (1984) 1; G. A. Miller, Int. Rev. Nucl. Phys. 1 (1984) 189.

18. W. Weise, International Review of Nucl. Phys., 1 (1984) 116; D. Robson, Topical Conference on Nuclear Chromodynamics, J. Qiu and D. Sivers (eds.), World Scientific, Singapore, 174 (1988).

19. D. I. Diakonov, V. Yu. Petrov, and P. V. Pobylitsa, Nucl. Phys. B306 (1988) 809.

20. S. Kahana, V. Soni, and G. Ripka, Nucl. Phys. A415 (1984) 351.

21. M. Birse and M. Banerjee, Phys. Rev. D31 (1985) 118.

22. C. V. Christov et al., Progr. Part. Nucl. Phys. 37 (1996) 91.

23. R. Alkofer and H. Reinhard, Chiral Quark Dynamics, Springer, 1995.

24. U. Vogl and W. Weise, Progr. Part. Nucl. Phys. 27 (1991) 195.

25. S. Coleman, J. Wess, and B. Zumino, Phys. Rev. 177 (1969) 2239.

26. S. Weinberg, Phys. Rev. Lett. 18 (1967) 188.

27. L. Ya. Glozman and E. I. Kuchina, Phys. Rev. C49 (1994) 1149.

28. L. Ya. Glozman, Z. Papp, and W. Plessas, Phys. Lett. B381 (1996) 311.

29. E. Witten, Nucl. Phys. B156 (1979) 269.

30. S. Coleman and E. Witten, Phys. Rev. Lett. 45 (1980) 100.

31. D. O. Riska, Phys. Reports 181 (1989) 207.

32. K. Dannbom, L. Ya. Glozman, C. Helminen, and D. O. Riska, in preparation.

33. D. Weingarten, Nucl. Phys. B (Proc. Suppl.) 34 (1994) 29.

34. T. D. Cohen and D. B. Leinweber, Comments Nucl. Part. Phys. 21 (1993) 137.

35. G. 't Hooft, Nucl. Phys. B72 (1974) 461.

36. K. F. Liu and S.-J. Dong, Quark Model from Lattice QCD, Preprint UK 194-03, hep-lat 9411067. 